\title{
SUPPORTING INFORMATION \\ Preferential End-to-End Assembly of Gold Nanorods by Biotin-Streptavidin Connectors
}

K. K. Caswell, James N. Wilson, ${ }^{\#}$ Uwe H. F. Bunz, ${ }^{* \#}$ and Catherine J. Murphy*

Department of Chemistry and Biochemistry, University of South Carolina, 631 Sumter

St., Columbia, SC 29208, USA

Scheme 1.

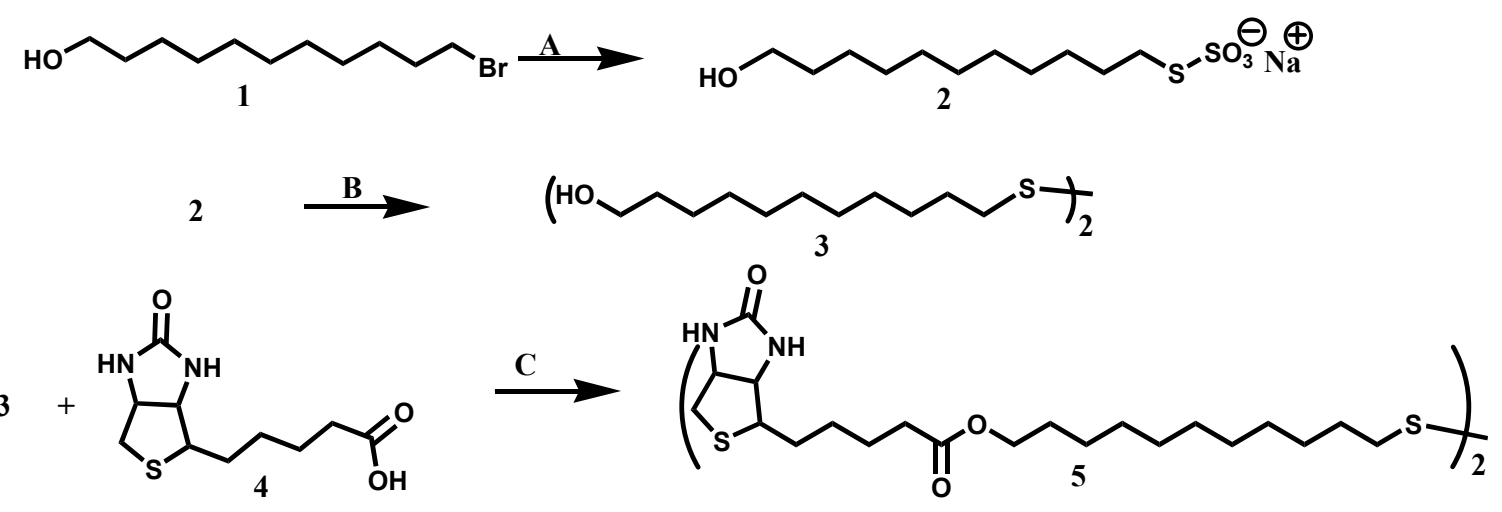

A. Bromo undecanol (5.23 g, $20.8 \mathrm{mmol})$ was dissolved in $25 \mathrm{~mL} \mathrm{MeOH}$ and $10 \mathrm{~mL}$ $\mathrm{H}_{2} \mathrm{O}$. The solution was heated to reflux and sodium thiosulfate $(5.00 \mathrm{~g}, 31.6 \mathrm{mmol})$ was added. After $6 \mathrm{~h}$ of refluxing, the reaction mixture was reduced to $6.43 \mathrm{~g}$ of a colorless powder, 2 , which was used directly in the subsequent reaction.

B. Bunte salt $2(6.43 \mathrm{~g}, 20.1 \mathrm{mmol})$ and thiourea $(1.60 \mathrm{~g}, 21.0 \mathrm{mmol})$ were combined in a $10 \% \mathrm{HCl}$ solution $(125 \mathrm{~mL})$. The mixture was heated to reflux for $4 \mathrm{~h}$, filtered and recrystallized from a hexane/chloroform mixture yielding $4.15 \mathrm{~g}$ of a pale yellow waxy solid 3.(yield 95\%). ${ }^{1} \mathrm{H} \mathrm{NMR}(\mathrm{CDCl} 3,300 \mathrm{MHz}): \delta 3.63\left(\mathrm{~m}, 4 \mathrm{H},-\mathrm{O}-\mathrm{CH}_{2}-\right), 2.66(\mathrm{t}$, $\left.\mathrm{J}_{3 \mathrm{H}, \mathrm{H}}=7.32 \mathrm{~Hz}, 4 \mathrm{H},-\mathrm{S}-\mathrm{CH}_{2}-\right), 1.63(\mathrm{~m}, 4 \mathrm{H}, \beta-\mathrm{OH}), 1.55(\mathrm{~m}, 4 \mathrm{H}, \beta-\mathrm{SH}), 1.13(\mathrm{bm}$, $28 \mathrm{H}) .{ }^{13} \mathrm{C} \mathrm{NMR}(\mathrm{CDCl} 3,100 \mathrm{MHz}): \delta \quad 63.30,39.42,33.05,29.81,29.71,29.65,29.44$, 28.74, 25.96. IR: $3357.8,3192.0,2918.1,2848.7,1469.7,1346.2,1319.2,1303.8$, 1269.1, 1242.1, 1211.2, 1186.1, 1134.1, 1076.2, 1062.7, 1037.6, 1014.5, 1002.9, 974.0, 717.5, 707.8, 601.7, 520.7. Mp: $56-59^{\circ} \mathrm{C}$.

C. 1,3-Dicyclohexylcarboiimide (2.73 mmol, $0.563 \mathrm{~g})$ was dissolved in dry DMF (5.0 $\mathrm{mL})$. Biotin $(2.05 \mathrm{mmol}, 0.500 \mathrm{~g}), 4$-(N,N-dimethylamino $)$ pyridine $(0.205 \mathrm{mmol}, 25.0$ 
$\mathrm{mg})$ and $3(0.683 \mathrm{mmol}, 0.277 \mathrm{~g})$ was added. The reaction was stirred at room temperate for $20 \mathrm{~h}$, then at $50{ }^{\circ} \mathrm{C}$ for $4 \mathrm{~h}$. The reaction was allowed to cool and the DMF was removed by vacuum distillation. $\mathbf{5}$ was obtained by this procedure:

The residue was stirred with dichloromethane and filtered to remove the excess biotin. The dichloromethane was removed by rotary-evaporator. The resulting solid was dissolved in $100 \mathrm{~mL}$ of methanol. Ice-cold water was added drop-wise until the appearance of a grayish solid which was filtered off and dried under vacuum producing $53.0 \mathrm{mg}$ of a pale yellow solid (yield $8.7 \%$ ).

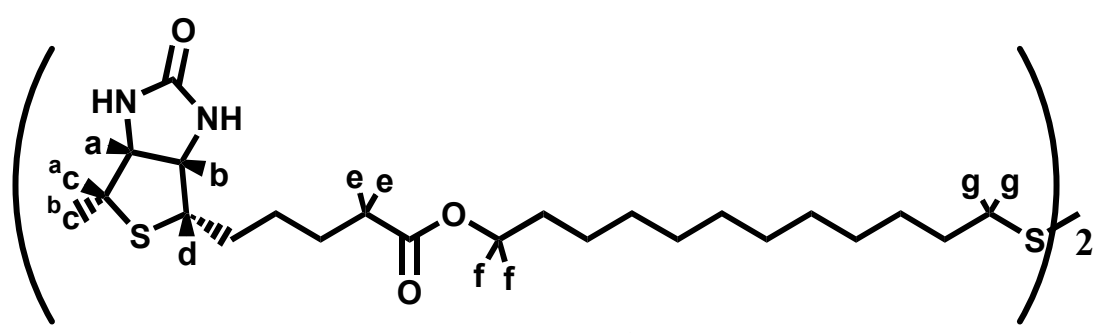

Figure 1. Assignment of ${ }^{1} \mathrm{H}$ NMR shifts.

${ }^{1} \mathrm{H}$ NMR (CDCl3, $\left.300 \mathrm{MHz}\right): \delta 5.70(\mathrm{~s}, 2 \mathrm{H}, \mathrm{N}-\mathrm{H}), 5.22(\mathrm{~s}, 2 \mathrm{H}, \mathrm{N}-\mathrm{H}), 4.49(\mathrm{~m}, 2 \mathrm{H}, \mathbf{b})$, $4.31(\mathrm{~m}, 2 \mathrm{H}, \mathbf{a}), 4.04\left(\mathrm{t}, 4 \mathrm{H}, \mathrm{J}_{3 \mathrm{H}, \mathrm{H}}=7.41 \mathrm{~Hz}, \mathbf{f}\right), 3.15(\mathrm{~m}, 2 \mathrm{H}, \mathbf{d}), 2.93\left(\mathrm{dd}, 2 \mathrm{H}, \mathrm{J}_{3 \mathrm{H}, \mathrm{H}}=\right.$ $\left.12.9 \mathrm{~Hz}, \mathrm{~J}_{4 \mathrm{H}, \mathrm{H}}=4.94 \mathrm{~Hz}, \mathbf{c}^{\mathbf{a}}\right), 2.74\left(\mathrm{~d}, 2 \mathrm{H}, \mathrm{J}_{3 \mathrm{H}, \mathrm{H}}=13.2 \mathrm{~Hz}, \mathbf{c}^{\mathbf{b}}\right), 2.66\left(\mathrm{t}, 4 \mathrm{H}, \mathrm{J}_{3 \mathrm{H}, \mathrm{H}}=\right.$ $7.14 \mathrm{~Hz}, \mathbf{g}), 2.31\left(\mathrm{t}, 4 \mathrm{H}, \mathrm{J}_{3 \mathrm{H}, \mathrm{H}}=7.41 \mathrm{~Hz}, \mathbf{e}\right), 1.63(\mathrm{~m}, 20 \mathrm{H}), 1.26(\mathrm{bm}, 28 \mathrm{H}) .{ }^{13} \mathrm{C} \mathrm{NMR}$ $(\mathrm{CDCl} 3,100 \mathrm{MHz}): \delta \quad 173.81,163.65,64.52,61.90,60.08,55.42,40.54,39.17,33.93$, 29.43, 29.16, 28.29, 28.44, 28.33, 28.22, 25.86, 24.82. IR: 3325.0, 2929.7, 2854.5, $1732.0,1654.8,1585.4,1569.9,1558.4,1485.1,1456.2,1434.9,1363.6,1303.8,1282.6$, 1245.9, 1188.1, 1174.6, 1139.9, 1116.7, 1101.3, 1026.1, 997.1, 873.7, 819.7, 756.0, 729.0, 705.9, 657.7, 640.3, 603.7, 507.2. Mp: 202-204 ${ }^{\circ} \mathrm{C}$ (dec.) MS (FAB) $\left(\mathrm{C}_{42} \mathrm{H}_{74} \mathrm{~N}_{4} \mathrm{O}_{6} \mathrm{~S}_{4}\right): m / z=859$. 